\title{
Implementación de un programa de mediación escolar: Análisis de las dificultades percibidas y propuestas de mejora $^{1}$
}

\section{Implementation of a school mediation program: analyzing the perceived difficulties and ways to improve it}

\author{
Rosa PULIDO VALERO, Sonsoles CALDERÓN-LÓPEZ, Gema MARTÍN-SEOANE \\ y Beatriz LUCAS-MOLINA \\ Universidad Nacional de Educación a Distancia, Universidad de Castilla La-Mancha, \\ Universidad Complutense de Madrid y Universidad de Valencia
}

Recibido: Marzo 2013

Aceptado: Mayo 2013

\begin{abstract}
Resumen
La investigación realizada analiza la puesta en marcha de un Equipo de Mediación en un centro de Secundaria de la Comunidad de Madrid, en concreto, la percepción de las dificultades encontradas por los participantes en dicho equipo. Durante un curso académico (2011-2012) se asistió a reuniones internas, actividades de formación y se realizaron grupos de discusión. Se han observado dificultades en diversos niveles (falta de implicación, dificultades de coordinación interna y con otros órganos, etc.), así como alta satisfacción con otros aspectos (posibilidades de mejora de la convivencia o habilidades personales adquiridas). Como conclusiones se presentan las propias sugerencias de mejora del grupo para el próximo curso, y, de cara a la implantación en otros centros, la importancia de la contextualización y la adopción de una perspectiva a largo plazo. Parece determinante que las personas que en él se impliquen sean capaces de priorizar objetivos a corto plazo y tener en mente un periodo mínimo de implementación de dos años, para poder avanzar en el proceso y no desanimarse ante las dificultades.
\end{abstract}

Palabras clave: convivencia escolar; intervención; mediación entre iguales; resolución de conflictos; prácticas restaurativas.

\footnotetext{
${ }^{1}$ El estudio presentado en este artículo fue realizado gracias al Premio de Investigación Aplicada de la Fundación Lafourcade Ponce concedido en 2011, para la elaboración del estudio sobre la implementación de programas de mediación en el contexto escolar y su relación con la promoción de una Psicología Positiva en el campo de la educación
} 


\begin{abstract}
This research study analyzes the implementation of a Mediation Program at a high school in Madrid. It focuses on the difficulties that the participants encountered while implementing it. During the school year 2011-2012, meetings and training sessions, as well as several focus groups discussions were carried out. Difficulties of different nature were observed (lack of involvement, internal and external coordination difficulties, among others aspects). On the other hand, the participants also expressed their satisfaction with the Mediation Program (as it facilitated a better coexistence in their school or the development of personal skills). Furthermore, some participants' suggestions to improve the Mediation Program are presented. Finally, the necessity of contextualizing these projects within a long term perspective is pointed out. It seems a key factor to be able to prioritize short term objectives, and to consider at least a period of two years for a successful implementation of the program and to carry on with the process in spite of difficulties.
\end{abstract}

Keywords: school coexistence; intervention; peer mediation; conflict resolution; restorative practices.

La mediación es una técnica de ayuda a personas enfrentadas para que entiendan su conflicto de una forma constructiva y busquen soluciones basadas en la colaboración. Una tercera persona ajena al conflicto, denominada mediador/a, facilita el análisis de lo ocurrido, a través de un proceso estructurado, con pasos y herramientas conocidos y manejados por dicha figura. Éste/a debe caracterizarse por su neutralidad e imparcialidad, ya que su función es asistir a las partes para que ellas mismas puedan tomar sus propias decisiones.

El origen de esta intervención suele situarse en culturas indígenas y grupos religiosos, que valoran especialmente el cuidado de las relaciones interpersonales en la vida en comunidad. Sus comienzos en el contexto legal se encuentran en 1974 en el proyecto Ontario-Canadá. En ella, dos jóvenes que habían causado daños en unas 22 propiedades fueron enviados a conversar con las víctimas y a llegar a un acuerdo para el pago de los daños; aunque, como reconoce el propio autor del marco teórico de la justicia restaurativa, los facilitadores de este primer encuentro se limitaron a decirles que llamaran a la puerta y se presentaran, que ellos estarían detrás esperando (Zehr, 2002, 2004).

Por el contrario, en cuanto a su origen en el contexto escolar existe cierta controversia. Más bien puede hablarse de teorías desarrolladas desde diferentes perspectivas (Pulido, Martín-Seoane y Lucas-Molina, 2013): a) las teorías de resolución de conflictos, aplicadas en 1987 en EEUU siguiendo el modelo de justicia restaurativa, en el denominado San Francisco's Community Board Program, y posteriormente en Canadá (Reimer, 2011; Vaandering, 2010), con especial énfasis en el hecho de que una conducta inadecuada puede no desaparecer tras una sanción (justicia retributiva), y que puede ser mucho más educativo ayudar a la persona a asumir su responsabilidad (justicia restaurativa); b) los diálogos y conferencias restaurativas, también como continuación del modelo judicial en Australia y Nueva 
Zelanda (Drewery, 2004; Drewery y Kecskemeti, 2011), aunque enfatizando más la importancia de la comunidad como apoyo al menor; c) el aprendizaje cooperativo reclamando un origen incluso previo al marco judicial, y aportando el matiz de la cooperación y colaboración de forma interdependiente (Johnson et al., 1995; Johnson y Johnson, 1989, 1995, 1996a, 1996b, 2001, 2004); d) los recursos de ayuda entre iguales, desarrollados principalmente en Gran Bretaña desde el año 2000 (Cowie, Hutson, Dawn y Myers, 2008; Cowie, Hutson, Oztug y Myers, 2008; Hutson y Cowie, 2007; Noaks y Noaks, 2009; Sellman, 2011), que incorporan la necesidad de promover redes de apoyo y de amistad. El concepto de prácticas restaurativas, especialmente utilizado en la literatura anglosajona (Bickmore, 2011; Cowie, 2010; Cremin, 2010; Kane et al., 2008, 2009; Macready, 2009; Morrison 2010) intenta aglutinar estas perspectivas, aunque existe un menor uso en nuestro país (Pulido, Martín-Seoane, Lucas-Molina y Calderón, en revisión-a).

En cuanto a la puesta en marcha de este tipo de programas en el contexto escolar en nuestro país, en primer lugar hay que señalar que tanto el Ministerio de Educación y Ciencia como diversas Consejerías destacan su interés para la mejora de la convivencia, como el "Libro Blanco de la Mediación en Cataluña" (Casanovas, Magre y Lauroba, 2011). A pesar de dichas iniciativas para promocionar su puesta en marcha, encontramos que esta se ha extendido de manera desigual. Por un lado, existe una gran variabilidad geográfica, siendo las variables más relevantes (Pulido, Martín-Seoane, Lucas-Molina y Calderón, en revisión-b): quién asume el rol de mediador/a, quién forma a los mediadores/as y cómo se lleva a cabo, desde qué aproximación teórica, y si es una implementación curricular, extracurricular o global. Teniendo en cuenta esto, es importante que el centro elija de manera flexible la alternativa que vaya más en sintonía con su realidad (Boqué, 2007), de modo que algunos autores proponen la necesidad de incorporar fases de evaluación previa para delimitar su situación concretas (Fernández, coord., 2007; Torrego, coord., 2006). Por otro lado, conviene señalar que la implementación de la mediación es aún escasa en nuestro país. En el estudio del Observatorio Estatal de Convivencia Escolar del Ministerio de Educación (Díaz-Aguado, Martínez Arias y Martín, 2010), se observó que, de los 278 equipos directivos evaluados, solamente el 23,7\% dicen disponer de un Equipo de Mediación (en lo sucesivo EM) y se plantean la necesidad de mejorarlo. Son escasos los estudios que evalúen de manera sistemática su implementación y niveles de eficacia. En la experiencia piloto desarrollada en Madrid entre 1998-2002, se observó una gran satisfacción en los 24 centros participantes, aunque un 58\% de ellos no llegaron a implementar el programa después de la formación, debido, entre otros factores, a la "dificultad para dar el impulso inicial" (Torrego y Galán, 2008, p. 384), tomando como fuente de información a los profesores y equipos directivos, sin contar con la visión del alumnado. En un estudio posterior (Boqué y García Raga, 2010), evaluando a 97 docentes de distintas etapas educativas en centros catalanes, observaron dificultades como la falta de tiempo (según un $41,03 \%$ ), de formación $(17,95 \%)$ y otros motivos $(15,38 \%)$, como la imposibilidad de resolver determinados conflictos mediante mediación, la falta de un plan de convivencia preventivo, el desánimo del profesorado 
por la invisibilidad de resultados a corto plazo, las tradiciones de la cultura del centro, etc. El 52,9\%\% que no puso en marcha un EM menciona motivos como cambios en el equipo directivo y en sus prioridades, la ausencia de conflictividad en el centro o la necesidad de nueva formación.

Teniendo en cuenta todo lo expuesto, el objetivo de esta investigación es estudiar la percepción de los participantes en la implementación de un programa de mediación escolar, analizando tanto las dificultades vividas en el proceso de implementación y su origen como las propuestas de mejora planteadas para superar las limitaciones. Este estudio permitirá conocer en mayor profundidad qué cuestiones subyacen al desánimo y a la dificultad para dar el impulso inicial, motivos ambos de abandono en la implementación de los programas, según las investigaciones previas. Asimismo, se pretende cubrir la laguna de información existente sobre estos procesos desde la perspectiva del alumnado.

\section{Método}

Se trata de un estudio de investigación-acción que, desde un planteamiento etnográfico, permite profundizar en la experiencia de los participantes. En dicho marco colaborativo, se propuso compartir ideas sobre su experiencia, así como debatir con ellos las respuestas que estaban dando o desearían darle a los problemas detectados. Se trata de un estudio descriptivo de caso único, por lo que los resultados no son generalizables a otras realidades distintas de la evaluada. A pesar de ello, puede ayudar a otros centros a entender la complejidad que suponen estos programas.

\section{Muestra}

La investigación se realizó en un instituto público de Enseñanza Secundaria de la Comunidad de Madrid, durante el curso escolar 2011-12. Dicho centro se ubicaba en un contexto socioeconómico medio, con un considerable porcentaje de alumnado de origen inmigrante y un gran tamaño (claustro superior a 100 profesores). Según indicaron los propios profesores, no era especialmente conflictivo ni tenía problemas de convivencia importantes.

Respecto al profesorado participante, se recogió información de tres profesores de Jefatura de Estudios (en lo sucesivo JE), cinco profesores que han participado en el EM y la orientadora. Todos los profesionales implicados tienen más de diez años de experiencia y se iniciaron en la mediación con un alto grado de motivación. Respecto al alumnado, de los 140 que participaron en la formación de cinco horas recibida el curso anterior, 40 decidieron ser miembros del EM y sólo 8 mostraron su interés por continuar un año después (principalmente debido a que en segundo de Bachillerato disponían de menos tiempo). Una vez realizada la campaña de captación en el curso 2011-12, se consiguió formar un grupo final de 19 personas con grandes diferencias en 
cuanto a su participación: un grupo de 9 alumnos/as han asistido a la formación con elevada periodicidad e interés, otro grupo de unos 10 alumnos/as ha asistido más irregularmente (aproximadamente un $50 \%$ de las actividades). La mayor parte del alumnado participante (un $75 \%$ ) eran mujeres y procedían, en su mayoría, de tercero y cuarto de ESO, aunque también había cuatro alumnos de bachillerato, tres de primero y uno de segundo curso.

\section{Técnicas de recogida de datos}

Se recogieron datos utilizando las siguientes técnicas:

a) Observaciones de las reuniones realizadas a lo largo del curso, tanto con los miembros del EM, como entre la coordinadora del EM, JE y orientación. Dichas reuniones solían tener una duración de entre 40 minutos y una hora aproximadamente.

b) Grupos de discusión con el profesorado y el alumnado formado durante este curso. Se han llevado a cabo seis sesiones, con una asistencia variable, en torno a una media de ocho personas (tres profesores y cinco alumnos en la mayor parte de los casos). Los temas planteados tenían que ver con las dificultades encontradas al asumir su nuevo rol. Consideramos este espacio distinto al anterior puesto que en las reuniones los temas y el orden de participación era abierto, mientras que para los grupos de discusión se seguía el guión propuesto por las investigadoras y se favorecía en el turno de palabra a aquellos que menos intervenían (generalmente, los alumnos/as). Estos grupos duraban aproximadamente 40 minutos y se desarrollaban en torno a tres temáticas: beneficios del programa, dificultades encontradas y formas de resolverlas, y principales aprendizajes.

\section{Procedimiento}

A continuación se describe tanto el procedimiento de implementación del EM como la evaluación llevada a cabo. El EM, como se ha explicado, durante el curso previo ya había recibido formación (de 5 horas de duración), tras la cual creó un buzón físico y una cuenta de correo electrónico para facilitar su localización al resto del alumnado, se diseñó un tríptico informativo y carteles que se colgaron por el centro, y llevaron a cabo tres mediaciones al final del curso en las que participaron como mediadores tanto profesorado como alumnado. A pesar de poder haberse considerado como un equipo ya establecido, debido a las limitaciones detectadas por los propios miembros del EM (falta de formación, escasez de participantes, etc.), fue necesario partir prácticamente de cero en el presente curso, distinguiéndose las siguientes fases:

1) Reuniones iniciales. Los cuatro profesores del EM que habían recibido formación el año anterior, los tres de JE y la orientadora se reunieron una o dos veces al mes, de octubre a diciembre, para analizar la situación. No se contó con la 
participación del alumnado hasta el mes de Diciembre, cuando sólo se presentó una única alumna, que apenas intervino en la reunión.

2) Se decidió realizar, en una segunda fase durante el mes de enero, la captación de más profesorado y alumnado, interesándose un único profesor (que no volvió a asistir), $\mathrm{y}$ de los 70 alumnos/as iniciales, sólo 19 se apuntaron al EM.

3) Para captar a familiares se incluyó información en la página web del AMPA, llamando un padre interesado, aunque finalmente no asistió a la formación por dificultades de horario.

4) En una cuarta fase se impartió un nuevo curso de formación en mediación, para todos los participantes, con una duración aproximada de 15 horas y con una periodicidad semanal entre los meses de febrero y mayo después del horario escolar (de 14 a 15 horas). Las decisiones sobre la formación se tomaron conjuntamente con el profesorado, en función de sus necesidades. Utilizando una combinación del material propuesto por Torrego (coord., 2000) y Boqué (2005), se trabajaron los siguientes módulos: a) El conflicto: tipos y procesos; b) Aproximación a la mediación como alternativa a la resolución de conflictos; c) Actitudes y técnicas de comunicación: verbal-no verbal, escucha activa, mensajes en primera persona, imparcialidad, reflejar sentimientos, errores típicos, etc.; d) Características de las entrevistas por separado: manejo de la curva de la ira, etc.; e) Características de los encuentros de mediación: fases, qué hacer y qué no hacer, etc. Siguiendo las variables mencionadas en la fundamentación teórica, propuestas por Pulido et al. (en revisión-a), la formación en el centro ha sido mixta (profesorado y alumnado), extracurricular, impartida por un profesional experimentado en mediación (ajeno al centro) y centrada en el enfoque de la resolución de conflictos. Respecto al hecho de que la formación la impartiera una persona ajena al centro, es necesario concretar dos aspectos: primero, que la persona que impartió la formación, a pesar de formar parte del equipo de investigación, siempre fue distinta de aquellas personas que llevaron a cabo la recogida de datos; y segundo, todo el proceso de implementación del programa se realizó de manera colaborativa entre investigadores-profesorado-estudiantes, sin ser en ningún caso puesto en marcha de manera directiva por el formador.

5) Desde el mes de abril, se celebraron paralelamente reuniones de los miembros del EM (alumnado y profesorado) para analizar dificultades, ya que algunos de ellos ya estaban mediando, y para fomentar la cohesión del grupo.

6) En cuanto a los casos de mediación que paralelamente se realizaron, no contamos con información al respecto, ya que no se permitió la presencia de investigadores ni su grabación, aunque había un adulto siempre presente observando. Dichas observaciones no serán tenidas en cuentas en este trabajo por su escasa fiabilidad.

7) Paralelamente a las fases anteriores, de abril a junio, se han llevado a cabo grupos de discusión con los miembros del EM, como un espacio independiente a sus 
reuniones, con el fin de recabar información sobre reflexiones. En los grupos participaron estudiantes y profesorado de manera conjunta.

Las transcripciones de los grupos de discusión fueron analizadas por separado por dos miembros del equipo investigador, fragmentándolas en unidades mínimas de significado y clasificándolas en categorías con contenido similar. Posteriormente, las dos investigadoras discutieron e interpretaron los datos categorizados, consensuando las discrepancias. De este modo, se ha llevado a cabo una triple estrategia de triangulación que hace posible el control de la validez interna y confirmabilidad, a semejanza de estudios previos sobre implementación de programas educativos (LlopisGoig et al., 2011): triangulación de técnicas (observación en reuniones, grupos de discusión), de responsables de la recogida de información y del análisis final de los datos, así como el trabajo colaborativo entre los cuatro miembros del equipo investigador para revisar el análisis, las interpretaciones y los resultados obtenidos.

\section{Resultados}

Para una presentación más organizada de los resultados, las categorías de análisis de las transcripciones se agruparán en dos bloques: dificultades y aspectos susceptibles de mejora; y puntos fuertes o aspectos positivos.

\section{Dificultades y aspectos susceptibles de mejora}

Entre los principales aspectos señalados por los participantes se encuentran:

- Número insuficiente de miembros

Se hizo patente desde el inicio que, a pesar de que el EM ya existía, se había producido un elevado número de bajas entre profesorado y alumnado. Cuando se realizaron reuniones para captar más interesados/as, las tasas de participación no fueron altas, aunque entraban dentro de lo que la coordinadora del equipo consideraba esperable. Una de sus explicaciones sobre el elevado número de alumnos/as que participaron en la sesión de información (140 el curso anterior y 70 el presente), y la muy reducida incorporación posterior al EM, fue que la sesión informativa se realizaba en horario lectivo. Cuando se les preguntó a los miembros del EM por los motivos de dicha falta de implicación, la principal razón tener que venir después de clase o perderse el recreo, sin ninguna compensación. Se mencionó la necesidad de reconocer de alguna forma su trabajo (con un diploma o acto público, en el caso del alumnado, o reconociéndolo como "horas de guardia", en el caso del profesorado). Respecto a la falta de interés del profesorado, un miembro del EM lo explicó así:

Yo creo que en cada clase debería ser obligatorio que hubiese un coordinador de mediación (...).Asistencia voluntaria, ino! Asistencia voluntaria para baloncesto o futbol, pero mediación, desde mi punto de 
vista, es tan interesante para el futuro social, que sería necesario que hubiese un chaval en clase que haya hecho el curso (Profesor mediador)

- Necesidad de formación antes de mediar

Algunos profesores que participaban desde el curso anterior señalaron que la formación inicial había sido insuficiente, por lo que se veían "muy verdes" y con necesidad de "refrescar". Como uno de los profesores sentenció: "no me quiero meter en charcos de los que no pueda salir". Respecto al alumnado, debido al escaso número de interesados/as, el centro no consideró obligatoria la asistencia, y permitió a todos continuar en el EM (aunque sólo un 50\% asistió de manera regular), generando una sensación de injusticia:

Yo quiero que haya gente en mediación, obviamente, pero yo creo que si tú te apuntas a algo tienes que tener un compromiso, y yo veo que aquí no se ha cumplido, y yo estoy un poco cabreada por eso. Porque yo creo que hay gente que se ha apuntado y no ha venido, y tienen casos y no han venido a la formación. Solo lo hacen para perder clase. Yo conozco a gente que ha venido a casi todas las sesiones de formación y no le han dado ningún caso y se ha cabreado y no viene (Alumna mediadora)

Aunque, tanto alumnado como profesorado que asistió a las sesiones insiste en la necesidad de dicha formación y de la práctica posterior.

Yo creo que eso es fundamental porque una persona que no ha tenido formación no creo que pueda mediar bien (Alumna mediadora)

La dificultad de hacer preguntas abiertas, de no juzgar y no proponer soluciones, que a base supongo de practicar... iQue también me parece difícil poder practicar mucho! (Profesor mediador)

La coordinadora del programa mencionó la necesidad, de cara al próximo año, de que la formación se realizara de manera intensiva (una semana, todas las tardes) al inicio de curso (frente a este, que se inició en Febrero), y que parte de dicha formación fuera impartida por el alumnado/profesorado formado este año, de modo que puedan transmitir lo aprendido.

- Problemas de coordinación interna

Este fue otro de los problemas más comentados, con aspectos como la no existencia de un listado de miembros del equipo o el hecho de que el aula de mediación estuviera en un lugar de difícil acceso. Una profesora lo explicaba de esta forma a principio de curso: 
Parece como si hubiera una gran dispersión de los datos, y los alumnos perciben ese caos (...) En ocasiones nos ha llevado hasta semanas localizar a los propios alumnos/as que se había decidido que mediaran y luego a los alumnos en conflicto (Profesora mediadora)

Se identificaron cuestiones básicas a resolver, llegando a solucionarse la elección de coordinador, la realización de reuniones periódicas y un lugar más visible para las reuniones. Otras, sin embargo, no fueron solventadas, como la toma de notas durante/después del proceso de mediación o la presencia de una persona de guardia, siempre localizable en el aula. Otro aspecto que se mencionó fue el mediar en una franja horaria diferente del recreo (para no privar del tiempo de descanso ni a los mediados ni a los mediadores), proponiendo de 14 a 15 horas o en tutoría (si el centro establecía un mismo horario para todos los grupos), aunque el profesorado lo veía poco viable debido al volumen de alumnos/as. Finalmente la coordinadora demandó la necesidad de algún apoyo, ya que en ocasiones se sentía desbordada.

- Problemas de coordinación con el resto de agentes educativos

Respecto a los problemas de coordinación con otros órganos del centro, algunas quejas se refieren a la falta de criterio para la derivación de casos, puesto que se habían recibido casos de JE que claramente no eran adecuados, como uno de acoso escolar y otro de enfrentamiento entre bandas. En el primero decidieron sentarse "a hablar" con las alumnas, para que acordaran unos "mínimos de convivencia", siendo conscientes de que la mediación no era la intervención más adecuada, y que sería insuficiente.

Por otro lado, los miembros del EM consideran que, después de lo aprendido en la formación, ahora son conscientes de que existen muchos más casos "mediables" en el centro.

Normalmente en el instituto hay muchísimos casos, porque lo sabemos, y es que no llegan por ninguna parte a mediación, porque no saben que lo pueden resolver. Por eso, si no llegan casos, debemos echarles una mano, para que nos hagan un poquito de caso (Alumna mediadora)

Creo que es importante que esto lo tengamos en cuenta porque como equipo creo que estamos cayendo en cierto vicio en considerar solo mediables aquellos conflictos que han llegado ya a un enfrentamiento, en parte porque todos nos llegan desde Jefatura. También tendríamos que empezar a plantearnos qué otros conflictos vemos que no se han detectado, o que no nos han pedido que intervengamos, pero que se pueden canalizar a través de mediación que podemos ofertar (Profesora mediadora) 
Otro problema de coordinación con JE es el riesgo de que si un alumno/a "cuenta lo que le ha pasado", además de "desvirtuar el proceso", también "duplica el trabajo", y puede suponer "cansancio si tiene que volver a contar todo al detalle al EM", e incluso llegar a "quemarles". Se han utilizado expresiones como "se corre el riesgo de convertirnos en una sucursal, y que los alumnos nos vean como una filial de Jefatura de Estudios", o el problema de que se demande al EM las "conclusiones" de las mediaciones (viéndose obligados a recordarles que es confidencial). Todas estas observaciones resultan llamativas cuando tres profesores de JE y la orientadora asistían a las reuniones, destacando continuamente la "necesidad de tener un protocolo de intervención claro", aunque esto nunca llegó a materializarse.

\section{- Dificultades en la difusión}

La toma de decisiones sobre cómo informar al resto del centro de su existencia ocupó buena parte del tiempo de las reuniones del EM. Algunas de las percepciones al respecto son:

Uno de los problemas del año pasado es que no se nos ha visto como el grupo de mediación (Profesor mediador)

Nadie sabe lo que es mediación. En el instituto muy poca gente lo sabe. Hay que hacer algo para eso (Alumna mediadora)

Finalmente se acordó, como prioridad para el curso siguiente ya que este no dio tiempo, que los propios alumnos/as mediadores/as se encargaran de informar a través de las tutorías, así como hacer un cartel con sus fotos para que pudieran ser identificados directamente.

\section{Puntos fuertes o aspectos positivos}

Se señalaron aspectos relacionados con los beneficios de este tipo de actuaciones o con su propia satisfacción personal con lo aprendido.

- Beneficios de tener un EM en el centro

El alumnado y el profesorado que se formó en mediación señalaron la importancia de contar con este recurso, por sus ventajas frente a otras actuaciones (es más eficaz, evita que el conflicto aumente en gravedad o se cronifique, ayuda a la maduración de los participantes), como se recoge a continuación:

Yo creo que los resuelve, si se consigue llegar al final, los resuelve de verdad. Hay otro tipo de soluciones que resuelven parcialmente, temporalmente. La mediación resuelve de verdad. Y desde la realidad del 
ser humano que es la convivencia. El conflicto no es malo, es real, es natural (Profesor mediador)

Puede que los resuelva más rápidamente (...) Si el conflicto va a la jefa de estudios, por ejemplo, hay un protocolo que seguir que conlleva más tiempo (...). La solución puede ser más traumática, de tipo disciplinario. (...) Hay años que ha habido con mucha frecuencia (refiriéndose a las sanciones disciplinarias) (Profesor mediador)

Quizás sea más automático ese procedimiento, si se puede hacer una entrevista con una parte y luego la otra entrevista con la otra (...) Pero bueno, en algún caso podría ser eso, que en tres sesiones, cuatro... en una semana el conflicto quedara en vías de extinción, porque eso ya depende de la persona claro (Profesor mediador)

Para lo que es la mediación, y para lo que yo creo que es buena, es porque a veces las personas solucionan los problemas entre ellas sin haberse escuchado y sin haberse puesto en la piel del otro (Alumna mediadora)

(...) el conflicto sigue larvado, sigue ahí (refiriéndose a si no lo hablas con alguien). Incluso puede empeorar. En ese tiempo en el que no dices nada puede que el conflicto vaya a más (Profesor mediador)

Actitud muy abierta porque en el conflicto del último día yo ahí no veía, no le encontraba sentido al proceso de mediación. Sin embargo, luego vi que, por lo menos es dar la oportunidad a que uno se explique y la otra parte se explique (...) El simple hecho de que alguien les escuche ya es mucho para ellos (Profesor mediador)

En diversas ocasiones se les preguntó si ellos acudirían al EM para resolver un conflicto propio. Aunque muchos se negaron rotundamente al principio y algunos tuvieron sus dudas incluso al final porque consideraban que les daría "vergüenza" hablar de sus cosas con los mediadores/as, en el último grupo de discusión algunos ya sí plantearon que podrían hacerlo:

Yo iría cuando no me viera capaz de solucionarlo, o valoro que no se puede solucionar, porque, o lo solucionamos regulín y al otro día también pensaría "aquí pasa algo" (Alumna mediadora)

Yo creo que intentar a ver si puedes solucionarlo por ti mismo, y si ves que el problema es que no puedes, pues también pueden ayudarte (Alumna mediadora) 
- Satisfacción con los conocimientos adquiridos

Los asistentes a la formación mostraron su satisfacción con el conocimiento adquirido y su utilidad. Se aplicó el cuestionario de valoración propuesto por Torrego (2000) y se obtuvo una elevada calificación. Por otro lado, a lo largo de la formación, alumnos/as y profesores/as fueron señalando aspectos que al principio no habían entendido pero que fueron comprendiendo al aplicarlos a casos reales. En varias ocasiones surgieron cuestiones que, a pesar de haberse trabajado en la formación, sólo fueron comprendidas al ponerlas en práctica. Por ejemplo, uno de los profesores, a pesar de haber recibido formación sobre la importancia de manejar la curva de la ira en las entrevistas por separado, después de una actividad de roleplaying comentó lo siguiente (cuando la persona expresó que había sentido que no había podido "desahogarse"):

Tampoco lo habiamos dicho antes..., que era uno de los objetivos por otra parte.... Parece muy bonita la palabra: desahogarse (Profesor mediador)

En una de las sesiones finales de formación, otra persona expresó que por fin había entendido cuál era el objetivo:

Mediación es para que te pregunten y te des cuenta de lo que de verdad hay. Los sentimientos que hay con la otra persona (Alumna mediadora)

Los mismos participantes sugirieron aspectos a mejorar en la formación, como por ejemplo: ampliarla a un mayor número de alumnos/as, profesorado, e incluso a personal no docente y familiares; que se realice al principio del curso y que participen en las sesiones teóricas también los miembros del EM, para que puedan transmitir la importancia de lo que han aprendido desde su propia experiencia personal.

Por último, hay que mencionar que, a pesar de que pueda parecer que las dificultades encontradas superan a los aspectos positivos, casi todos los miembros del EM consideraron que había merecido la pena el esfuerzo realizado este año, y estaban dispuestos a continuar en el curso siguiente. De hecho, en las últimas reuniones ya se estaba comentando cómo incorporar nuevos miembros, qué alumnos/as podrían impartir parte de la formación, así como retomar la difusión del $\mathrm{EM}$ al iniciar las clases.

\section{Resumen de las propuestas de los mediadores para mejorar el programa}

En relación a las aportaciones realizadas por los propios miembros del EM destacan las siguientes: la participación obligatoria de algún alumno/a de todas las clases, un mayor número de profesoras/es implicados; mayor exigencia en la asistencia a la formación para poder actuar como mediador/a; necesidad de un reconocimiento a todos/as por parte del centro y al profesorado como horas de guardia; realizar la formación al principio del curso, en horario intensivo de tarde, e impartido en parte por los propios 
miembros del EM; crear un protocolo de coordinación con el resto de profesionales del centro; realizar las mediaciones después del horario de clases; llevar un registro interno de los casos y establecer guardias; y retomar a principio de curso la campaña de difusión.

\section{Conclusiones}

Del análisis de los resultados obtenidos se derivan una serie de conclusiones que se presentan a continuación y que consideramos que debería valorar cualquier centro educativo interesado en poner en marcha este tipo de programas:

1) La implementación de este tipo de programas es lenta y deben establecerse de modo muy preciso objetivos a corto plazo, para evitar abandonos prematuros.

Como ya se ha comentado, a pesar de que ya existía un EM formado, que contaba incluso con tríptico y carteles informativos, fue necesario prácticamente partir de cero: captando a más profesorado y alumnado, nueva formación, aspectos organizativos nuevos que iban surgiendo, etc. Todo ello apunta a la necesidad de considerar la implementación como un proceso a largo plazo, donde se secuencien los objetivos priorizando algunos para el primer curso y dejando otros para más adelante (segundo, tercer o incluso cuarto curso de implementación). Además, se ha observado la necesidad de que exista tanto una persona que coordine el EM como distintas comisiones de trabajo para distribuir las tareas a realizar.

2) Las prioridades deben establecerse considerando la realidad de cada centro.

Como puede observarse en el caso presentado, el EM había recibido formación en mediación, elaborado un tríptico y un cartel, y creado un buzón y una cuenta de correo electrónico para recibir los casos de mediación, pasos considerados típicos en la creación de un EM (Torrego y Villaoslada, 2004). Sin embargo, sin la nueva formación ofertada al equipo existente que permitió relanzar el proyecto, quizás hubiera acabado desapareciendo. Es importante señalar que cada centro debe intentar abordar los pasos que se vea capaz de acometer. Por ejemplo, puede necesitar dedicar un año exclusivamente a la formación, sin realizar difusión y sin empezar a mediar hasta que se considere que el grupo está consolidado. Por todo ello, estamos totalmente de acuerdo con la idea de respetar la "potestad de cada centro para decidir cómo quiere incorporar la mediación, cuándo desea iniciar la formación, qué nivel de implicación le resulta más conveniente, cuánto tiempo quiere dedicar a ello, o qué lugar le corresponderá dentro del currículum" (Boqué, 2007, p. 62). En este sentido, cabe plantear como cuestión para la reflexión si fue adecuada la decisión de iniciar las mediaciones durante el curso pasado tras un proceso de formación tan corto. Sin embargo, de no haber sido así, ¿habría llegado el EM al momento en que se encuentra ahora? 


\section{3) Es necesario dar protagonismo en el EM a representantes de todos los sectores}

En el proceso estudiado se ha observado un aumento de la implicación del alumnado, que pasó de no hablar en las reuniones iniciales a sentirse capaz de impartir la formación en el curso siguiente. Este aspecto es de gran relevancia ya que son un elemento clave a la hora de que este tipo de programas se consoliden en los centros. En la misma línea sería interesante trabajar con familias y con personal no docente. En estudios previos se menciona la importancia de que el coordinador sea una persona muy implicada en el mismo, que dinamice y coordine adecuadamente (Torrego y Galán, 2008), a lo que añadiríamos que sería ideal que hubiese más de un coordinador, o varios sub-coordinadores especializados en tareas (difusión, formación, revisión de casos, etc.) y que, además, estos representaran a todos los grupos participantes. Por último, también sería interesante incluir a alumnos de primer ciclo de la ESO, aunque todavía no actúen como mediadores al no estar, probablemente, preparados para ello desde un punto de vista madurativo. De este modo, sin embargo, conseguiríamos implicarles de cara a cursos sucesivos, cuando el vínculo con el centro de los mediadores ya expertos vaya desapareciendo.

Respecto a las aportaciones del estudio es necesario resaltar la importancia de este tipo de trabajos que describen detalladamente las realidades de los centros educativos cuando se enfrentan a tareas complejas como la que aquí se ha presentado. Es imprescindible identificar qué aspectos contribuyen a que este tipo de intervenciones resulten exitosas, o de qué variables puede depender que se conviertan en un fracaso. En este sentido debemos beneficiarnos de las descripciones que los propios participantes ofrecen del proceso vivido y de cómo creen que ellos pueden (o no) solucionarlo. Como otros autores ya han mencionado previamente (Cambra y Palou, 2007), refiriéndose a experiencias de innovación en otras áreas, es a través de este análisis como podremos llegar a entender las inquietudes, los dilemas y las creencias de los protagonistas y realizar estudios que les permitan de verdad mejorar sus realidades.

Finalmente, estudios como este son necesarios para también analizar qué aportan este tipo de programas desde el punto de vista de la cultura de centro. En este sentido es necesario destacar la necesidad de que sean proyectos consensuados por la comunidad educativa, la importancia de superar modelos autoritarios en favor de la participación y la responsabilización de los estudiantes, o la importancia de una cultura de aprendizaje en el centro. El proceso de investigación-acción llevado a cabo ha permitido implicar a los estudiantes y al profesorado en un dialogo crítico sobre los conflictos que se producen en su centro educativo y la manera de resolverlos, generando lo que Freire (1970) denomina ciclos de reflexión-acción sobre dichos problemas, capaces de facilitar que los implicados sean conscientes, se sientan con poder de cambio en la situación, propiciando la necesaria democratización del sistema. 


\section{Referencias bibliográficas}

BICKMORE, K. (2011). Location, location, location: restorative (educative) practices in classrooms, documento presentado al ESRC en los Seminarios de Justicia Restaurativa: Restorative Approaches to Conflict in schools, Seminar 4, University of Cambridge. Recuperado de:

http://www.educ.cam.ac.uk/research/projects/restorativeapproaches/seminarfour/

BOQUÉ, C. (2005). Tiempo de mediación. Taller de formación de mediadores y mediadoras en el ámbito educativo. Barcelona: Ceac educación.

BOQUÉ, C. (2007). Guía de mediación escolar. Barcelona: Octaedro.

BOQUÉ, C. y García Raga, L. (2010). Evaluación diferida de la formación del profesorado en convivencia y mediación, Revista Electrónica Interuniversitaria de Formación del Profesorado, 13(3), 87-94.

CAMBRA, M Y PALOU, J. (2007). Creencias, representaciones y saberes de los profesores de lenguas en las nuevas situaciones plurilingües escolares de Cataluña. Cultura y Educación, 19 (2), 149-163.

CASANOVAS, P., MAGRE, J. Y LAUROBA, M.L. (2011). El Libro Blanco de la Mediación en Cataluña. Departamento de Justicia de la Generalitat de Cataluña. Recuperado de: http://www.llibreblancmediacio.com/phpfiles/public/libroBlancoDownloadCounter.p hp

COWIE, H., HUTSON, N., DAWN, J., Y MYERS, A. M. (2008). Taking stock of violence in U.K. Schools risk, regulation, and responsibility. Education and Urban Society, 40(4), 494-505.

COWIE, H., HUTSON, N., OZTUG, O. Y MYERS, C. (2008). The impact of peer support schemes on pupils' perceptions of bullying, aggression and safety at school. Emotional and Behavioural Difficulties, 13(1), 63 - 71.

COWIE, H. (2010). Restorative practice in school: a psychological perspective, documento presentado al ESRC en los Seminarios de Justicia Restaurativa: Interdisciplinary perspectives on restorative approaches to reducing conflicts in schools: exploring theory and practice from cross-national and international settings, Seminar 3, University of Cambridge. Recuperado de:

http://www.justicereparatrice.org/www.restorativejustice.org/10fulltext/cowiehelen/v iew

CREMIN, H. (2010). RJ into schools: does it go? Some theoretical and practical considerations, documento presentado al ESRC en los Seminarios de Justicia Restaurativa: Inter-disciplinary perspectives on restorative approaches to reducing conflicts in schools: exploring theory and practice from cross-national and international settings, Seminar 1, University of Cambridge. Recuperado de: 
http://www.educ.cam.ac.uk/research/projects/restorativeapproaches/seminarone

DÍAZ-AGUADO, M. J., MARTÍNEZ ARIAS R. Y MARTÍN, J. (2010). Estudio Estatal Sobre La Convivencia Escolar En La Educación Secundaria Obligatoria, Ministerio de Educación. Recuperado de:

https://sede.educacion.gob.es/publiventa/detalle.action?cod=13567

DREWERY, W. (2004). Conferencing in schools: punishment, restorative justice, and the productive importance of the process of conversation. Journal of Community \& Applied Social Psychology, 14, 332-344.

DREWERY, W. Y KECSKEMETI, M. (2011). Restorative Practice and Behaviour Management In Schools: Discipline Meets Care. Waikato Journal of Education 15(3), 101-114.

FERNÁNDEZ, I. (Coord., 2007). Prevención de la violencia y resolución de conflictos. El clima escolar como factor de calidad. Madrid: Narcea.

FREIRE (1970). Pedagogía del oprimido. México: Siglo XXI.

HUTSON, N. Y COWIE, H. (2007). Setting up an email peer support scheme, Pastoral Care, December, 12-16.

JOHNSON, D.W. Y JOHNSON, R. (1989). Cooperation and competition: Theory and research. Edina, MN: Interaction Book Company.

JOHNSON, D.W., JOHNSON, R., DUDLEY, B., WARD, M. Y MAGNUSON, D. (1995). The impact of peer mediation training on the management of school and home conflicts. American Educational Research Journal, 32(4), 829-844.

JOHNSON, D.W. Y JOHNSON, R. (1995). Creative controversy: Intellectual challenge in the classroom (3rd ed.). Edina, MN: Interaction Book Company.

JOHNSON, D.W. Y JOHNSON, R. (1996a). Conflict resolution and peer mediation programs in elementary and secondary schools: a review of the research. Review of Educational Research, 66(4), 459-506.

JOHNSON, D.W. Y JOHNSON, R. (1996b). Teaching all students how to manage conflicts constructively: the peacemakers program. The Journal of Negro Education, 65(3), 322-335.

JOHNSON, D.W. Y JOHNSON, R. (2001). Peer mediation in an inner-city elementary school. Urban Education, 36, 165.

JOHNSON, D.W. Y JOHNSON, R. (2004). Implementing the "Teaching Students To Be Peacemakers Program". Theory into Practice, 43, 68-79.

KANE, J., LLOYD, G., MCCLUSKEY, G., RIDDELL, S., STEAD, J. Y WEEDON, E. (2008.) Collaborative evaluation: balancing rigor and relevance in a research study of restorative approaches in schools in Scotland. International Journal of Research \& Method in Education, 31(2), 99-111. 
KANE, J., LLOYD, G. MCCLUSKEY, G., MAGUIRE, R., RIDDELL, S., STEAD, J. Y WEEDON, E. (2009).Generating an inclusive ethos? Exploring the impact of restorative practices in Scottish schools. International Journal of Inclusive Education, 13(3), 231-251.

LLOPIS-GOIG, R., ESCARTI, A., PASCUAL, C., GUTIÉRREZ M. Y MARÍN, D. (2011). Fortalezas, dificultades y aspectos susceptibles de mejora en la aplicación de un Programa de Responsabilidad Personal y Social en Educación Física. Una evaluación a partir de las percepciones de sus implementadotes. Cultura y Educación, 23(3), 445-461.

MACREADY, T. (2009). Learning social responsibility in schools: a restorative practice. Educational Psycholy in Practice. 25(3), 211-220.

MORRISON, B. (2010). Beyond the bad apple: analytical and theoretical perspectives on the development of restorative approaches in schools, documento presentado al ESRC en los Seminarios de Justicia Restaurativa: Inter-disciplinary perspectives on restorative approaches to reducing conflicts in schools: exploring theory and practice from cross-national and international settings, Seminar 1, University of Cambridge. Recuperado de:

http://www.educ.cam.ac.uk/research/projects/restorativeapproaches/seminarone

NOAKS, J. Y NOAKS, L. (2009). School-based peer mediation as a strategy for social inclusion. Pastoral Care in Education, 27(1), 53-61. DOI: 10.1080/02643940902731880

PULIDO, R., MARTÍN-SEOANE, G. Y LUCAS-MOLINA, B. (2013). Orígenes de los Programas de Mediación Escolar: Distintos enfoques que influyen en esta práctica restaurativa. Anales de Psicología, 29(2), 385-392. DOI: 10.6018/analesps.29.2.132601 http://dx.doi.org/10.6018/analesps.29.2.132601

PULIDO, R., MARTÍN-SEOANE, G., LUCAS-MOLINA, B. Y CALDERÓN, S. (en revisión-a). Análisis de la Implementación de los Programas de Mediación Escolar: Posibilidades y Limitaciones.

PULIDO, R., MARTÍN-SEOANE, G., LUCAS-MOLINA, B. Y CALDERÓN, S. (en revisión-b). Principios y Prácticas Restaurativas para mejorar la Convivencia Escolar: La necesidad de cuidar las Relaciones Interpersonales.

REIMER, K. (2011). An exploration of the implementation of Restorative justice in an Ontario public school. Canadian Journal of Educational Administration and Policy, 119, 1-42.

SELLMAN, E. (2011). Peer mediation services for conflict resolution in schools: what transformations in activity characterize successful implementation? British Educational Research Journal, 37(1), 45-60. DOI: 10.1080/01411920903419992

TORREGO, J.C. (Coord.) (2000). Mediación de conflictos en instituciones educativas: Manual para la formación de mediadores. Madrid: Narcea. 
TORREGO, J.C. (Coord.) (2006). Modelo integrado de mejora de la convivencia. Estrategias de mediación y tratamiento de conflictos. Barcelona: Graó.

TORREGO, J.C. y Villaoslada, E. (2004). Modelo integrado de regulación de la convivencia y tratamiento de conflictos. Tabanque, Revista Pedagógica, 18, 31-48.

TORREGO, J.C. Y GALÁN, A. (2008). Investigación evaluativa sobre el programa de mediación de conflictos en centros escolares. Revista de Educación, 347, 369-394.

Vaandering, D. (2010). The significance of critical theory for restorative justice in education. The Review of Education, Pedagogy, and Cultural Studies, 32, 145-176. DOI: $10.1080 / 10714411003799165$

ZEHR, H. (2002). The Little Book of Restorative Justice. Pennsylvania: Good Books.

ZEHR, H. (2004). Commentary: Restorative justice: beyond victim-offender mediation. Conflict Resolution Quarterly, 22, 305-315.

\section{Correspondencia con los autores}

Rosa PULIDO VALERO

Departamento de Psicología Evolutiva y de la Educación

Universidad Nacional de Educación a Distancia

Juan del Rosal, 10, 28040, Madrid

e-mail: rpulido@psi.uned.es

\section{Sonsoles CALDERON-LOPEZ}

Departamento de Psicologia

Universidad de Castilla-La Mancha

Avda. Real Fabrica de Sedas, s/n

45600 Talavera de la Reina

Toledo

e-mail: sonsoles.Calderon@uclm.es

Gema MARTÍN SEOANE

Departamento de Psicología Evolutiva y de la Educación, UCM

Campus de Somosaguas. Pozuelo de Alarcón 28223, Madrid

e-mail: gema.martin@psi.ucm.es

Beatriz LUCAS-MOLINA

Departamento de Psicología Evolutiva y de la Educación, Universidad de Valencia

Avenida de Blasco Ibáñez, 21, 46010, Valencia

e-mail: beatriz.lucas@uv.es 